\title{
Self-Organizing-Maps en el estudio del concreto de alto rendimiento
}

\section{Self-Organizing-Maps in the study of high performance concrete}

\author{
Isabel Moromi Nakata ${ }^{1}$, Ana Torre Carrillo", Luis Acuña Pinaud², Francisco García Fernández ${ }^{3}$, Pedro \\ Espinoza Haro²
}

${ }^{1}$ Facultad de Ingeniería Civil, Universidad Nacional de Ingeniería. Av. Túpac Amaru, 210. Lima 25, Perú

${ }^{2}$ Facultad de Ingeniería Industrial y de Sistemas. Universidad Nacional de Ingeniería. Av. Túpac Amaru, 210.

Lima 25, Perú

${ }^{3}$ Dpto. Ingeniería Forestal. Universidad Politécnica de Madrid. Ciudad Universitaria S/N, 28040 Madrid,

España

DOI: https://doi.org/10.33017/RevECIPeru2014.0011/

\section{Resumen}

La fabricación de este tipo de concreto, se realiza tomando en cuenta la selección y caracterización de los materiales para elaborar un concreto de alta resistencia a la compresión y diseños de mezcla adecuados para estos fines. Los materiales empleados son el cemento hidráulico Portland Tipo I. Los agregados fino y grueso. Se sigue para el diseño de mezcla métodos indicados en el ACI 211.4R-93. Se han incorporado las microsílices y los aditivos superplastificantes que pueden reducir la demanda de agua y el contenido de cemento y pueden producir concretos con baja relación agua-cemento, alta resistencia y trabajabilidad normal o alta.

De otro lado se tiene el uso de las redes neuronales, denominadas Self-Organizing-Maps (RNSOM) que son redes no supervisadas con una sola capa. El objetivo en esta parte del trabajo es crear un grupo de RNSOM que permitan formar grupos o clústeres de probetas a partir de las variables de fabricación, sin involucrar la resistencia ni la carga de rotura de las probetas y contrastar estos resultados con los grupos de probetas de mayor resistencia o de menor resistencia, con tiempos y diseños que resulten óptimos.

Descriptores: Concreto de alta resistencia, microsílice, redes neuronales, base de datos

\section{Abstract}

The manufacture of this type of concrete is carried out taking into account the selection and characterization of materials to produce a high concrete compressive strength and mix designs suitable for these purposes. The materials used are hydraulic Portland cement Type I, fine and coarse aggregates. It follows the design of mixed methods in ACl 211.4R-93.

Be added the microsílices, also superplasticizers additives which may reduce demand for water and cement content and can produce concrete with low water-cement ratio, high strength and normal or high workability.

On the other hand there is the use of neural networks called Self-Organizing-Maps (RNSOM) that are not supervised by a single layer networks. The objective in this part of the work is to create an enabling RNSOM form groups or clusters of samples from manufacturing variables without involving resistance and tensile strength 
of the specimens and compare these results with larger groups of probes lower resistance or resistance with time and designs that are optimal.

Keywords: High-strength concrete, microsilica, neural networks, database

\section{Introducción}

El presente trabajo parte de una dualidad indesligable: la base de datos y las redes neuronales artificiales.

\subsection{La base de datos}

La base de datos (BD) fue elaborada por el Laboratorio de Ensayo de Materiales de la Facultad de Ingeniería Civil de la UNI (LEM-FIC-UNI). En una primera entrega, que es la que se empleará, se tiene 22 registros con los valores de las variables de fabricación de 296 probetas de concreto y un registro con los valores de la resistencia a la compresión.

En la elaboración de esta BD se tomaron en cuenta los siguientes objetivos: selección y caracterización de los materiales para elaborar un concreto de alta resistencia a la compresión y diseños de mezcla adecuados para estos fines.

Entre los materiales empleados está el cemento hidráulico Portland Tipo I. Una de las características más importantes de estos cementos hidráulicos es que fraguan y endurecen por la reacción química con el agua, e incluso, pueden endurecer bajo el agua. El agregado fino cumple con los requerimientos de la ASTM C 33. La gradación óptima de agregado fino para el concreto de alta resistencia es determinada más por su efecto en la demanda de agua. El agregado grueso juega un rol crítico en el concreto de alta resistencia pues las propiedades mecánicas del agregado grueso van a tener un efecto más pronunciado que cuando son usados en el concreto normal. Para el diseño de mezcla (que van numeradas del 1 al 7) se usó el método indicado en el ACI 211.4R93. Se han incorporado las microsílices siguiendo las normas ASTM C 1240 y NTP 334.087. Los aditivos superplastificantes son aditivos reductores de agua de alto rango que obedecen las normas ASTM C494 y NTP 334.088. En la ASTM C 494 corresponden a los tipos $F$ (reductor de agua) y $G$ (reductor de agua y retardador de fraguado). Estos aditivos pueden reducir grandemente la demanda de agua y el contenido de cemento y pueden producir concretos con baja relación agua-cemento, alta resistencia y trabajabilidad normal o alta. En esta investigación el aditivo a usar es el PLASTOL 5000.

\subsection{Las redes neuronales}

Las redes neuronales elegidas para agrupar las probetas de concreto son las denominadas SelfOrganizing-Maps (RNSOM) que son redes neuronales no supervisadas que tienen solo una capa.

El objetivo en esta parte del trabajo es crear un grupo de RNSOM que permitan formar grupos o clústeres de probetas con ciertas características, como puede ser las de mayor resistencia o las de menor resistencia, con tiempos y diseños óptimos.

Sin embargo el problema de clasificar las probetas de máxima resistencia en una base de datos donde se conocen sus valores, es un problema trivial, porque bastará ordenar la resistencia de menor a mayor juntamente con los demás datos y esto nos dirá con todo detalle cuáles son las probetas de máxima y mínima resistencia y sus correspondientes características. Pero este resultado es irrelevante por cuanto no se estaría respondiendo al problema de la caracterización de las probetas de máxima resistencia en forma consistente, pero si se hace una clasificación con la misma base de datos, sin tomar en cuenta la resistencia y la carga de rotura y si la RNSOM logra agrupar las probetas de máxima resistencia, estaríamos frente a un modelo matemático que es capaz de decirnos cuáles son las características del concreto de máxima resistencia sin conocer a priori su valor.

\subsection{Las topologías de una capa de neuronas}

Las neuronas en la capa de una RNSOM pueden ser representadas por puntos o posiciones de un plano bidimensional $X Y$, formando los vértices de un grafo que son las posiciones de los pesos de las neuronas y las aristas o arcos los enlaces entre estos. 


\subsection{Las nociones de distancia entre neuronas}

Desde el punto de vista matemático existen infinitas nociones de distancia, una clase de ellas son las distancias $L_{p}$ (Minkowski) que se definen como sigue: Si $x \square\left(x_{1}, x_{2}, \ldots \ldots, x_{N}\right)$ y $y \square\left(y_{1}, y_{2}, \ldots \ldots, y_{N}\right)$ son dos vectores de $\square^{n}$, la distancia entre ellas, en el sentido de los $L_{p}$ se denota y define como:

$d_{p}(x, y) \square^{\square} \square \square\left|x_{i} \square y_{i}\right|^{\square^{\square}} \square$ donde $10 p \square \square$ es

$$
\square_{i \square 1} \quad \square \text { cualquier }
$$

número real.

Cuando $p \square 1$ la distancia se llama de Mahattan y coincide con la distancia que existe entre dos puntos de una ciudad cuyas calles forman una malla rectangular. Cuando $p \square 2$, se tiene la distancia euclideana, que es la longitud del segmento que une estos puntos. Cuando $p$ tiende al infinito la distancia $d_{p}(x, y)$ en el límite se convierte en la distancia del máximo y es $d_{\square}(x, y) \square \max \left|x_{i} \square y_{i}\right|$

$$
i \square 1,2, . . N
$$

(Chebychev). Existen otras nociones de distancia que vienen de otro tipo de conceptos, como la del enlace.

\section{RNSOM con diferentes topologías, distancias y variables de fabricación}

\begin{tabular}{|l|c|c|c|c|}
\hline $\begin{array}{c}\text { Característica } \\
\text { s de las } \\
\text { RNSOM }\end{array}$ & $\begin{array}{c}\text { RNSOM } \\
1\end{array}$ & $\begin{array}{c}\text { RNSOM } \\
2\end{array}$ & $\begin{array}{c}\text { RNSOM } \\
3\end{array}$ & $\begin{array}{c}\text { RNSOM } \\
4\end{array}$ \\
\hline no de clusters & 5 & 4 & 5 & 6 \\
\hline neuronas & {$[23]=6$} & {$[23]=6$} & {$[23]=6$} & {$[23]=6$} \\
\hline topologia & Gritop & Gritop & Gritop & Gritop \\
\hline metrica & Boxdist & Boxdist & Mandist & dist \\
\hline variables & $\begin{array}{c}14910 \\
12]\end{array}$ & {$[14910]$} & Todas & {$[1213]$} \\
\hline max. resisten. & $\begin{array}{c}96.04 \% \\
\text { min. resisiten. }\end{array} 99.04 \%$ & $95.46 \%$ & $98.91 \%$ & $98.41 \%$ \\
\hline $\begin{array}{l}\text { № de probeta } \\
\text { Max }\end{array}$ & 74 & 69 & 48 & 40 \\
\hline $\begin{array}{l}\text { № probeta en } \\
\text { el Clúster Min }\end{array}$ & 91 & 92 & 38 & 92 \\
\hline
\end{tabular}

Los resultados de los ensayos realizados con diferentes arquitecturas de RNSOM, con diferentes grupos de variables, en las que se eliminaron la resistencia y la carga de rotura se muestran en la tabla adjunta.

Esta indica que de las cuatro redes que se probaron y las diversas variables consideradas, la que dio mejores resultados de agrupamiento es la RNSOM2, por que las variables de entrenamiento no involucran la resistencia ni la carga de rotura. Los clústeres Max y Min de probetas de mayor y mínima resistencia, son más numerosas y con alto nivel de correlación con los datos reales.

\section{Conclusiones}

Las características de la RNSOM2 son: tiene [2 3]=6 neuronas, la topología es el de las mallas (gridtop), la distancia es la del máximo (boxdist) y el número de iteraciones para su entrenamiento fue de 1000. La base de datos para su entrenamiento comprende únicamente las variables: edad, cemento, micro sílice y arena. Luego del entrenamiento de la red con estas 4 variables, se han generado 4 clústeres: Clúster 1 con 44 probetas, Clúster 2 con 92 probetas, Clúster 5 con 91 probetas, Clúster 6 con 69 probetas.

\subsection{El Clúster 6}

Contiene a las probetas con resistencias altas correspondientes al primer tercio superior y guarda una correlación del $95.46 \%$ con las 69 probetas de la base de datos de mayor resistencia. Los diseños de este grupo de probetas son el 1 y 2 . Las edades son de 28 y 56 días. La cantidad de cemento empleada son los 2 niveles más altos, esto es 6 y 7, en una escala que va del 1 al 7 .

\subsection{El Clúster 2}

Contiene las probetas de resistencias menores del primer tercio inferior, con una correlación del $98.40 \%$ con las 92 probetas de la base de datos de menor resistencia. Las edades son 7 días. Los diseños son 4, 5, 6 y 7. La cantidad de cemento es de los más bajos o sea 1, 2, 3 y 4 .

Finalmente: La RNSOM2 juntamente con la base de datos formada por las 4 variables: edad, cemento, 
micro sílice y arena permiten llegar a clasificaciones muy similares a las de una BD con los valores de la resistencia y la carga de rotura de las probetas. Esto indica que estas 4 variables son determinantes en la fabricación del concreto de alto rendimiento.

\section{Agradecimientos}

El presente trabajo es parte del proyecto de investigación "Redes Neuronales y Simulación de Monte Carlo para el estudio del concreto de alta resistencia", financiado por CONCYTEC, según contrato: 137-FINCyT-IA-2013, que ejecutan las Facultades de Ingeniería Civil e Ingeniería Industrial y de Sistemas, de la Universidad Nacional de Ingeniería.

\section{Referencias}

[1] Oral E. L, Oral M. Predicting construction crew productivity by using Self Organizing Maps.

Automation in Construction. 19(6)(2010) 791-797.

[2] Marengo E. Bobba M. Robotti E. Liparota M. Modeling of the Polluting Emissions from a Cement Production Plant by Partial LeastSquares, Principal Component Regression, and Artificial Neural Networks. Environmental Science \& Technology. 40(1)(2006)272-280. [3] Nataraja M. C., Jayaram M. A, Ravikumar C.

N. Kohonen's feature maps for fly ash categorization, International Journal of Neural Systems, 16 (6)(2006) 457-466
[4] H. Adeli, Neural networks in civil engineering:1989-2000, Computer-Aided Civil and Infrastructural Engineering 16 (2001) 126-142

[5] Y. Cal, Soil classification by neural network, Advances in Engineering Software, 22 (1995) 95-97.

[6] M. D. A. Thomas, M. H. Shehata and S. G. Shashiprakash, The use of fly ash in concrete: Classification by composition, Cement, Concrete and Aggregates 21(2)(1995) 105-10.

[7] M. C. Nataraja, M. A. Jayaram and C. N. Ravikumar, Group-indexing fly ashes through radial basis function networks, Indian Concrete Journal 80(7) (2006) 39-45.

E-mail: imoromi@yahoo.com 